\title{
Cambio postincendio en la estructura y composición del estrato arbóreo y carga de combustibles en un bosque de Pinus douglasiana de México
}

\section{Post-fire change in structure and composition of the tree stratum and fuel load in a Pinus douglasiana forest in Mexico}

\author{
Shatya Devi Quintero-Gradilla", Enrique José Jardel-Peláez', Ramón Cuevas-Guzmán!, \\ Felipe García-Oliva² y Angelina Martínez-Yrizar ${ }^{3}$
}

\begin{abstract}
Universidad de Guadalajara. Centro Universitario de la Costa Sur. Departamento de Ecología y Recursos Naturales. Autlán de Navarro, Jalisco, México.
\end{abstract}

2 Universidad Nacional Autónoma de México. Instituto de Investigaciones en Ecosistemas y Sustentabilidad. Morelia, Michoacán. México.
3 Universidad Nacional Autónoma de México. Instituto de Ecología. Hermosillo, Sonora,

* Autor de correspondencia. shatya@yahoo.com

\section{RESUMEN}

En los bosques de pino con un régimen histórico de incendios superficiales frecuentes de baja severidad, bajo condiciones extremas del estado del tiempo o a consecuencia de la supresión del fuego, la severidad de los incendios puede incrementar. La respuesta de la vegetación a eventos de incendios severos se estudió en una cronosecuencia postincendio de 8 años, 28 años y 60 años en un bosque dominado por Pinus douglasiana en México. La densidad de árboles con diámetro $>2.5 \mathrm{~cm}$ y su área basal alcanzaron valores de 1095 árboles ha-1 \pm 609 árboles ha-1 y $10.0 \mathrm{~m}^{2} \mathrm{ha}^{-1} \pm 2.2 \mathrm{~m}^{2} \mathrm{ha}^{-1}$ a los 8 años; a los 28 años el área basal $\left(44.6 \mathrm{~m}^{2} \mathrm{ha}^{-1} \pm 3.0 \mathrm{~m}^{2} \mathrm{ha}^{-1}\right)$ fue cercana a la de los rodales de 60 años $\left(49.0 \mathrm{~m}^{2} \mathrm{ha}^{-1} \pm 6.4 \mathrm{~m}^{2} \mathrm{ha}^{-1}\right)$. Los pinos mantuvieron el mayor valor de importancia con el tiempo postincendio, se observó la formación de un subdosel de latifoliadas tolerantes a la sombra y un aumento en la riqueza y diversidad de especies; también se documentó que periodos largos sin incendios incrementan la carga de combustibles superficiales ligeros (66.0 Mg $\mathrm{ha}^{-1} \pm 5.5 \mathrm{Mg} \mathrm{ha}^{-1}$ en los sitios de 28 años y $61.5 \mathrm{Mg} \mathrm{ha}^{-1} \pm 4.6 \mathrm{Mg}^{-1}$ en los sitios de 60 años) y modifican el comportamiento del fuego (velocidad de propagación de $1.3 \mathrm{~m} \mathrm{~s}^{-1}$ a $4.1 \mathrm{~m} \mathrm{~s}^{-1}$ y la longitud de la llama de $0.7 \mathrm{~m}$ a $1.7 \mathrm{~m}$ entre los rodales de 8 años y los de 28 años y 60 años combinados). El manejo de los bosques de pino debe considerar el balance entre objetivos de conservación biológica y mitigación del peligro de incendios de alta severidad.

PALABRAS CLAVE: bosques mixtos de pino-latifoliadas; combustibles forestales; diversidad de especies; incendios severos; regímenes de incendios; Reserva de la Biosfera Sierra de Manantlán

\section{ABSTRACT}

In pine forests with an historical fire regime of frequent low severity surface fires, under extreme weather conditions or a consequence of fire suppression, the severity of fires may increase. Response of vegetation to severe fire events was studied in a chronosequence of 8 years, 28 years and 60 years in a forest dominated by Pinus douglasiana in Mexico. Tree density ( $>2.5 \mathrm{~cm}$ diameter) reached values of 1095 stems ha-1 \pm 609 stems ha-1 and a basal area of $10.0 \mathrm{~m}^{2} \mathrm{ha}^{-1} \pm 2.2 \mathrm{~m}^{2} \mathrm{ha}^{-1}$ in the 8 years stands; 28 years after stand-replacement fires, basal area $\left(44.6 \mathrm{~m}^{2} \mathrm{ha}^{-1} \pm 3.0 \mathrm{~m}^{2} \mathrm{ha}^{-1}\right)$ was close to that of 60 -year-old stands $\left(49.0 \mathrm{~m}^{2} \mathrm{ha}^{-1} \pm 6.4 \mathrm{~m}^{2}\right.$ ha-1). Pines showed the highest importance value across the chronosequence; with post-fire time, a formation of a sub-canopy of shade-tolerant broad-leaved species and an increase in species richness and diversity was observed. Long periods without fire increased surface fuel loading (66.0 $\mathrm{Mg}^{-1} \pm$ $5.5 \mathrm{Mg} \mathrm{ha}^{-1}$ for 28 years and $61.5 \mathrm{Mg} \mathrm{ha}^{-1} \pm 4.6 \mathrm{Mg} \mathrm{ha}^{-1}$ for 60 years-old stands) and changed fire behavior (spread velocity increased from $1.3 \mathrm{~m} \mathrm{~s}^{-1}$ to $4.1 \mathrm{~m} \mathrm{~s}^{-1}$ and flame length from $0.7 \mathrm{~m}$ to $1.7 \mathrm{~m}$ between the 8 years stands and 28 years - 60 years stands combined). Results suggest that the fire suppression policy in pine forests should be evaluated considering the balance between biological conservation goals and mitigation of high severity fire danger.

KEYWORDS: mixed pine-hardwood forests; species diversity; forest fuels; fire regimes; stand-replacement fires; Sierra de Manantlán Biosphere Reserve 


\section{INTRODUCCIÓN}

Los ecosistemas forestales juegan un papel clave en la regulación del ciclo global del carbono y su conservación, manejo sustentable y restauración constituyen componentes centrales de las medidas de mitigación del cambio climático (Schulze, Wirth y Heimann, 2000). En este contexto, el conocimiento de los efectos de los incendios forestales sobre la dinámica de los ecosistemas es un tema de estudio fundamental para el desarrollo de mejores prácticas de manejo del fuego, especialmente en el caso de bosques en los que este agente ha sido parte de su dinámica natural o histórica (Bowman et al., 2013), como es el caso de la mayor parte de los bosques de pino del mundo (Agee, 1998).

Dado que los efectos del fuego a escala del paisaje no son homogéneos, debido a la variación en la estructura de la vegetación y el complejo de combustibles, el tiempo transcurrido desde el último incendio, las condiciones topográficas y el estado del tiempo atmosférico durante estos eventos (Agee, 1993; Odion et al., 2014); se produce un mosaico heterogéneo de parches que mantienen distintas condiciones de hábitat en el paisaje y permiten la coexistencia de especies con distintos requerimientos ambientales y diferentes respuestas al fuego (Odion et al., 2014).

Los incendios forestales son un disturbio ecológico común en los bosques de pino de México, donde se encuentran 49 especies de Pinus $(40 \%)$ de las aproximadamente 120 en el mundo (Gernandt y Pérez-de la Rosa, 2014) y la conservación de estos bosques es importante por la diversidad de su biota, sus servicios ecosistémicos y su producción forestal (Galicia, Potvin y Messier, 2015). Los bosques de pino de las zonas templadas húmedas y sequía estacional cubren 6,15 millones de hectáreas en México (38\% de la superficie de bosques de pino del país) y presentan un régimen histórico de incendios superficiales frecuentes (intervalo de retorno de incendios de 2 años a 20 años a escala de rodales) y de baja severidad (Rodríguez-Trejo y Fulé 2003; Jardel-Peláez, PérezSalicrup, Alvarado-Celestino y Morfin-Ríos, 2014; Cerano-
Paredes et al., 2015). Bajo este régimen, la mayoría de los incendios tienen severidad baja, mientras que incendios forestales con una severidad moderada a alta ocurren en una proporción menor (Agee, 1993). Los incendios de baja severidad no modifican sustancialmente la cobertura del dosel y el fuego solo consume parte del mantillo sobre el suelo y la vegetación del sotobosque; los pinos dominantes y las especies de plantas asociadas a estos son resistentes o tolerantes al fuego (Rodríguez-Trejo y Fulé, 2003).

La supresión del fuego provoca con el tiempo el aumento de la vulnerabilidad de estos bosques a incendios más intensos, severos y difíciles de controlar, debido a la acumulación de combustibles y cambios en la estructura y composición de la vegetación (Pyne, Andrews y Laven, 1996). Por lo anterior, conocer su capacidad de recuperación en términos de estructura y composición de especies, es una cuestión relevante en el manejo de los bosques de pino y el diseño de mejores prácticas de manejo del fuego orientadas con objetivos de conservación biológica, producción silvícola, restauración ecológica o mitigación de emisiones de carbono.

Los incendios de alta severidad provocan una alta mortandad del arbolado del dosel y el subdosel (70\% $100 \%$, el consumo de toda o una gran parte de la materia orgánica sobre el suelo y el reinicio del rodal con una nueva cohorte dominada por especies de la sucesión temprana (Agee, 1993). La capacidad para recuperarse en términos de estructura y composición de especies después de incendios severos depende de la mortandad causada por el fuego y los cambios subsecuentes en la composición, densidad y distribución de tamaños de los árboles, así como de la posterior regeneración sobre la cual influye el tamaño de los claros abiertos por el incendio (Keyser, Lentil, Smith y Shepperd, 2008). La variación inherente en dichos factores hace más difícil identificar un patrón único de respuesta de la vegetación a la severidad de los incendios.

Los bosques de pino dominados por Pinus douglasiana Martínez en la Reserva de la Biosfera Sierra de Manantlán en México, se caracterizan por incendios frecuentes, superficiales y de baja severidad (Jardel-Peláez, 1991; 
Cerano-Paredes et al., 2015). En estos bosques, el alargamiento del intervalo sin fuego por más de 20 años llevó a la acumulación de altas cargas de combustibles superficiales (Alvarado et al., 2008) y a la ocurrencia de incendios de severidad mixta en los años 1983 y 2003 (Jardel-Peláez, 1991; 2008). Por lo anterior, es necesario conocer la respuesta de la vegetación a condiciones infrecuentes o extremas del régimen histórico de incendios; por ejemplo, cuando se presentan incendios severos, de reemplazo de rodales que reinician la sucesión.

\section{OBJETIVOS}

Los objetivos de este estudio fueron: 1) analizar cómo cambia la estructura y la composición de especies arbóreas con la edad del rodal después del incendio y 2) cuantificar el cambio en la carga de combustibles superficiales con el tiempo después del incendio. La hipótesis es que la estructura se hará más compleja y la carga de combustibles superficial se incrementará con el tiempo después del incendio.

\section{MATERIALES Y MÉTODOS}

\section{Área de estudio}

La Estación Científica Las Joyas (ECLJ) forma parte de la Reserva de la Biosfera Sierra de Manantlán (RBSM), localizada en el centro-occidente de México (19³4'14"$19^{\circ} 37^{\prime} 30^{\prime \prime}$ N y 104¹4'49"-104º1 $18^{\prime} 16^{\prime \prime}$ O); cubre una superficie de $1245 \mathrm{ha}$, en un gradiente elevacional entre los 1500 m y $2242 \mathrm{~m}$ snm (Fig. 1). El clima se caracteriza por una temperatura media anual de $15.5{ }^{\circ} \mathrm{C} \pm 0.2^{\circ} \mathrm{C}$, con precipitación media anual de $1826 \mathrm{~mm} \pm 94 \mathrm{~mm}$ y un cociente de evapotranspiración potencial/precipitación anual de 0.5. El régimen de lluvias es de verano (junioseptiembre), con precipitaciones ocasionales en invierno y un periodo de sequía en primavera que favorece la propagación de incendios entre marzo y principios de junio. La cubierta vegetal corresponde a bosque de pino-encino, bosque mixto de pino-latifoliadas, bosque mesófilo de montaña y matorrales secundarios, que cubren respectivamente $45 \%, 32 \%, 18 \%$ y $5 \%$ de la superficie del área de estudio (Cuevas-Guzmán y Jardel-Peláez, 2004).

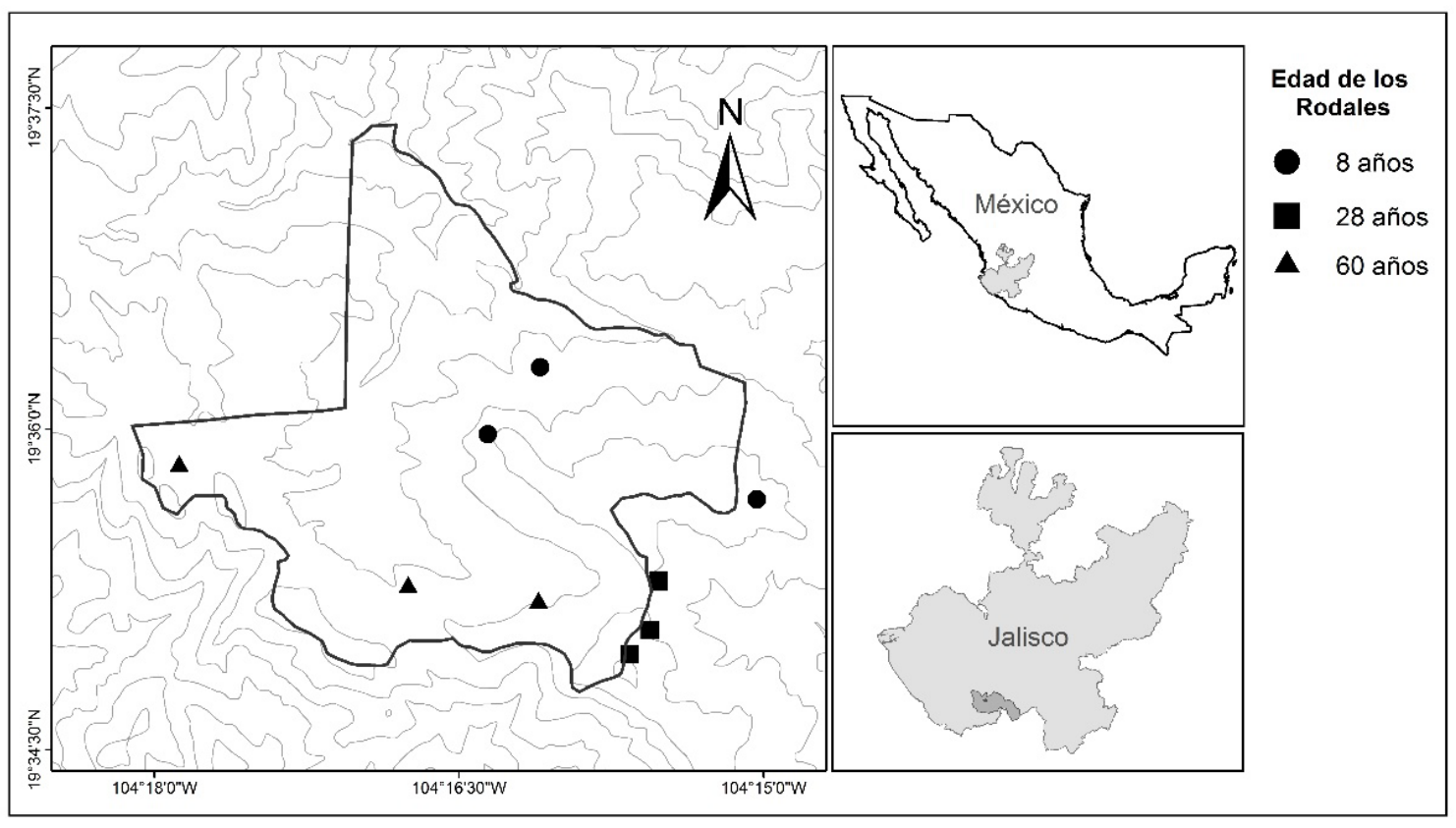

FIGURA 1. Localización de los rodales en la cronosecuencia postincendio de bosques dominados por Pinus douglasiana en el centro-occidente de México. 
Estudios basados en la datación dendrocronológica de incendios, indican que el régimen histórico de incendios en los bosques de pino de la ECLJ, y sus alrededores, se caracteriza por incendios frecuentes, superficiales y de baja severidad, con un intervalo medio de retorno de 2 años a 12 años (Jardel-Peláez, 1991; Cerano-Paredes et al., 2015). En el manejo de la ECLJ se ha suprimido el fuego con fines de restauración del bosque mesófilo de montaña y bosque mixto de pino-latifoliadas (Cuevas-Guzmán y Jardel-Peláez, 2004). El alargamiento del intervalo entre incendios por más de 20 años incrementó la carga de combustibles superficiales (49 $\mathrm{Mg} \mathrm{ha}^{-1}-96 \mathrm{Mg} \mathrm{ha}^{-1}$; Alvarado et al. 2008). Bajo estas condiciones, incendios de severidad mixta se presentaron en la ECLJ en 1983 y 2003 (Jardel-Peláez, 1991; 2008) lo que dio como resultado un patrón heterogéneo de parches de vegetación con afectaciones de severidad baja, media y alta. Los parches con afectaciones de alta severidad tuvieron una extensión de 2 ha a 40 ha, en los cuáles el fuego superficial de alta intensidad causó alta mortalidad (> 70\%), consumió la capa del mantillo y promovió el remplazo del rodal con el inicio de la sucesión secundaria.

Los sitios de estudio (Fig. 1) se establecieron en los parches con afectaciones de alta severidad en los incendios de 1983 y 2003, 8 años y 28 años después del último disturbio ecológico; una tercera condición seleccionada como referencia, corresponde a rodales con aproximadamente 60 años de edad establecidos después de intervenciones intensivas de corta de madera, seguidas de incendios. Estos rodales tienen la mayor edad promedio en el área de estudio y en ellos el fuego ha estado excluido por más de 30 años (Jardel-Peláez, 2008).

\section{Diseño del muestreo y métodos de campo}

Para evaluar el cambio en la estructura, incluyendo la diversidad de especies y composición del estrato arbóreo del bosque de pino después de incendios de remplazo de rodal, se compararon tres condiciones en una cronosecuencia de 8 años, 28 años y 60 años de edad. El enfoque de cronosecuencia, se basa en el supuesto de que la variación principal entre sitios se debe a la diferencia en el tiempo después del último disturbio ecológico. Para minimizar el efecto de las condiciones de sitio, éstos se establecieron en rodales con dominancia de $P$. douglasiana (> 70\% del área basal), ubicados en la misma zona bioclimática entre $1950 \mathrm{~m}$ y $2150 \mathrm{~m}$ snm y en la misma unidad geomorfoedafológica (Alfisoles sobre pórfido basáltico, en laderas medias con pendientes moderadas a fuertes). Se utilizó un diseño de muestreo anidado; para cada edad fueron seleccionados tres rodales independientes, separados espacialmente entre sí por una distancia entre $0.5 \mathrm{~km}$ y $2.4 \mathrm{~km}$. En el centro de cada uno de los rodales, evitando condiciones de borde, se establecieron de manera sistemática tres parcelas circulares de $500 \mathrm{~m}^{2}$ (0.05 ha) cada una, con una separación de $50 \mathrm{~m}$ entre sus centros. En estas parcelas se midió con cinta diamétrica el diámetro $(\mathrm{cm})$ a $1.30 \mathrm{~m}$ arriba del suelo $\left(\mathrm{D}_{1.30}\right)$ y la altura total $(\mathrm{m})$ con clinómetro, de todos los árboles vivos y muertos con diámetro $\geq 2.5 \mathrm{~cm}$. Los individuos se identificaron a nivel de especie.

La capa de hojarasca sobre el suelo o mantillo se diferenció en hojarasca fresca (HF) que mantiene su estructura y hojarasca fragmentada en descomposición (HD), y su carga $\left(\mathrm{Mg} \mathrm{ha}^{-1}\right)$ se estimó a partir de mediciones de profundidad y densidad aparente (Morfín-Ríos, JardelPeláez, Michel-Fuentes y Alvarado-Celestino, 2012). La profundidad de estas capas fue medida en 16 puntos por parcela, cada $3 \mathrm{~m}$ a lo largo de cuatro líneas radiales de 12.6 $\mathrm{m}$, partiendo del centro de las parcelas circulares. La densidad aparente de ambas capas se estimó midiendo su profundidad y colectando muestras de hojarasca en cuadros de $30 \mathrm{~cm} \times 30 \mathrm{~cm}$ en tres puntos establecidos al azar dentro de las parcelas circulares; estas muestras fueron secadas a $60{ }^{\circ} \mathrm{C}$ y pesadas en el laboratorio. El material leñoso caído fue cuantificado en tres líneas de $20 \mathrm{~m}$, separadas entre sí por un ángulo de $120^{\circ}$ partiendo desde el centro de la parcela circular, siguiendo el método de intersecciones planares de van Wagner (1982). 


\section{Análisis de datos}

Se cuantificaron los valores promedio de densidad de tallos (árboles ha $\left.\mathrm{a}^{-1}\right)$, área basal $\left(\mathrm{m}^{2} \mathrm{ha}^{-1}\right)$ de árboles vivos $\mathrm{y}$ muertos, carga $\left(\mathrm{Mg} \mathrm{ha}^{-1}\right)$ de hojarasca (HF y HD) y material leñoso caído por rodal. Se realizaron análisis de varianza (Anava), probando los supuestos de normalidad y homocedasticidad con las pruebas Kolmogorov-Smirnov y Levene, respectivamente. La estructura de diámetros y alturas se analizó a través de histogramas de frecuencia de árboles por categoría de tamaño. Los análisis estadísticos se realizaron con el programa InfoStat (Di Rienzo et al., 2008).

El índice de valor de importancia de las especies se estimó como el promedio de los valores relativos de abundancia, frecuencia y área basal. La riqueza de especies acumulada esperada se comparó con un método de rarefacción (Magurran, 2004) usando el programa EstimateS (versión 7.5). Los índices de diversidad de Simpson (1-D) y Shannon $(H)$ calculados con transformación $l n$, no cumplieron con el supuesto de homocedasticidad de varianzas, por lo que se analizaron por medio del método no paramétrico de bootstrap estimando los intervalos de confianza a 95\%, usando 250 repeticiones, con el programa InfoStat (Di Rienzo et al., 2008). Las diferencias significativas entre categorías de edad de los rodales fueron inferidas al observar que no hubiera traslape en los intervalos de confianza. La diversidad $\beta$ se calculó con el índice cuantitativo de Sørensen, basado en la abundancia de las especies de árboles (Magurran, 2004) en las tres condiciones de estudio con el programa PC-ORD 6.0 (McCune y Mefford, 2006).

\section{RESULTADOS}

\section{Estructura de rodales}

El cambio observado en la estructura de los rodales a través de la cronosecuencia mostró un patrón caracterizado por: (a) una rápida regeneración de los rodales en los claros abiertos por el fuego, cuya área basal alcanzó a los 28 años un valor cercano al de los rodales de 60 años; (b) la disminución a través del tiempo de la densidad de tallos de pino, aparejada con el aumento de su área basal, y (c) el incremento de la densidad y área basal de las especies latifoliadas (Fig. 2).

Los árboles de pino pasaron de $77.5 \%$ en los rodales en la etapa de regeneración o establecimiento ( 8 años), a $47.9 \%$ en la clase de edad de 28 años y se redujo a $10 \%$ en los rodales de 60 años de edad, pero en las tres clases de edad su área basal fue siempre mayor, pasando de $92 \%$ a los 8 años a $96 \%$ a los 28 años y disminuyendo a $81 \%$ a los 60 años (Fig. 2).

Estos cambios están relacionados con la variación observada en la distribución de tamaños de los árboles (Fig. 3). En los rodales de 8 años, $94 \%$ de los árboles ( $76 \%$ pinos y $18 \%$ latifoliadas) se concentró en las categorías de diámetro menores a $10 \mathrm{~cm}$, que corresponden a la regeneración establecida en los claros abiertos por los incendios; los árboles con diámetros mayores a $30 \mathrm{~cm}$ fueron los que sobrevivieron a los incendios del año 2003.

En los rodales de 28 años, la distribución de diámetros de los pinos mostró una forma de campana, la mayor parte de los tallos se concentró en categorías de diámetro intermedias de $20 \mathrm{~cm}$ a $45 \mathrm{~cm}$, que corresponden a árboles jóvenes establecidos después del incendio de 1983. La distribución de diámetros muestra una discontinuidad después de la clase de $50 \mathrm{~cm}$ y el grupo de árboles de mayor tamaño, representa a los árboles que sobrevivieron al incendio. En los rodales con 60 años de edad, los diámetros de la población de pinos mostraron también una distribución en forma de campana, con una base amplia entre los $30 \mathrm{~cm}$ y $70 \mathrm{~cm}$ de diámetro y la mayor frecuencia de los árboles concentrada en la categoría de $50 \mathrm{~cm}$ (Fig. 3). En contraste, la distribución de diámetros de las especies latifoliadas en los rodales de 28 años y 60 años de edad presentó una curva que tiende a una forma de "J invertida" y los árboles se concentran en las clases menores a $25 \mathrm{~cm}$ de diámetro.

La distribución de diámetros de las latifoliadas, comparada con su distribución de alturas (Fig. 3), muestra la formación de un estrato arbóreo bajo o subdosel, conforme aumenta el tiempo postincendio. En los rodales de 8 años de edad, 96\% de los árboles se ubicaron en las 
clases de altura entre $5 \mathrm{~m}$ y $10 \mathrm{~m}$ dominadas por los pinos, mientras que en los rodales de 28 años se observaron dos estratos: uno bajo (subdosel) con una altura promedio $\leq 10$ $\mathrm{m}$, formado por latifoliadas y uno alto (dosel) de $20 \mathrm{~m}$ a 30 $m$ de altura, dominado por pinos. En los rodales de 60 años de edad, hay también dos estratos definidos: el subdosel de latifoliadas (altura $\leq 20 \mathrm{~m}, 89 \%$ del total de árboles) y el dosel dominado por pinos ( $25 \mathrm{~m}$ a $45 \mathrm{~m}$ de altura, $11 \%$ del total de árboles).
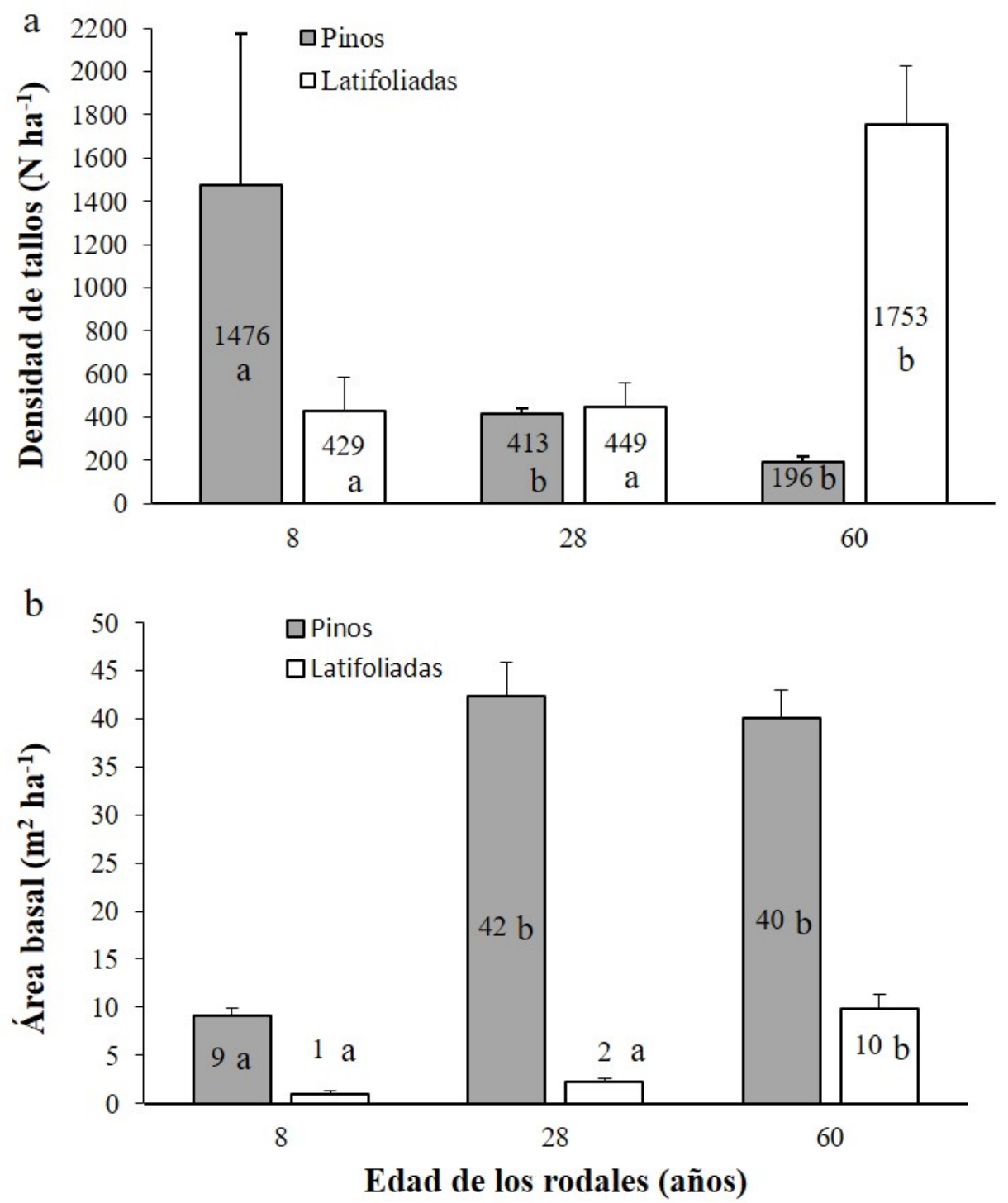

Figura 2. Densidad (a) y área basal (b) de los árboles (tallos con $D_{1.30} \geq 2.5 \mathrm{~cm}$ ) en la cronosecuencia postincendio de bosques dominados por Pinus douglasiana en el centro-occidente de México.

Las barras con letras diferentes muestran diferencia significativa $(P<0.05)$ de acuerdo con una prueba de Tukey. Las diferencias fueron estadísticamente significativas para la densidad de pinos $\left(F_{2,26}=6.3 ; P=0.006\right)$ y latifoliadas $\left(F_{2,26}=\right.$ 32.2; $P<0.001)$ y el área basal de pinos $\left(F_{2,26}=29.9 ; P<0.001\right)$ y latifoliadas $\left(F_{2,26}=28.4 ; P<0.001\right)$. 

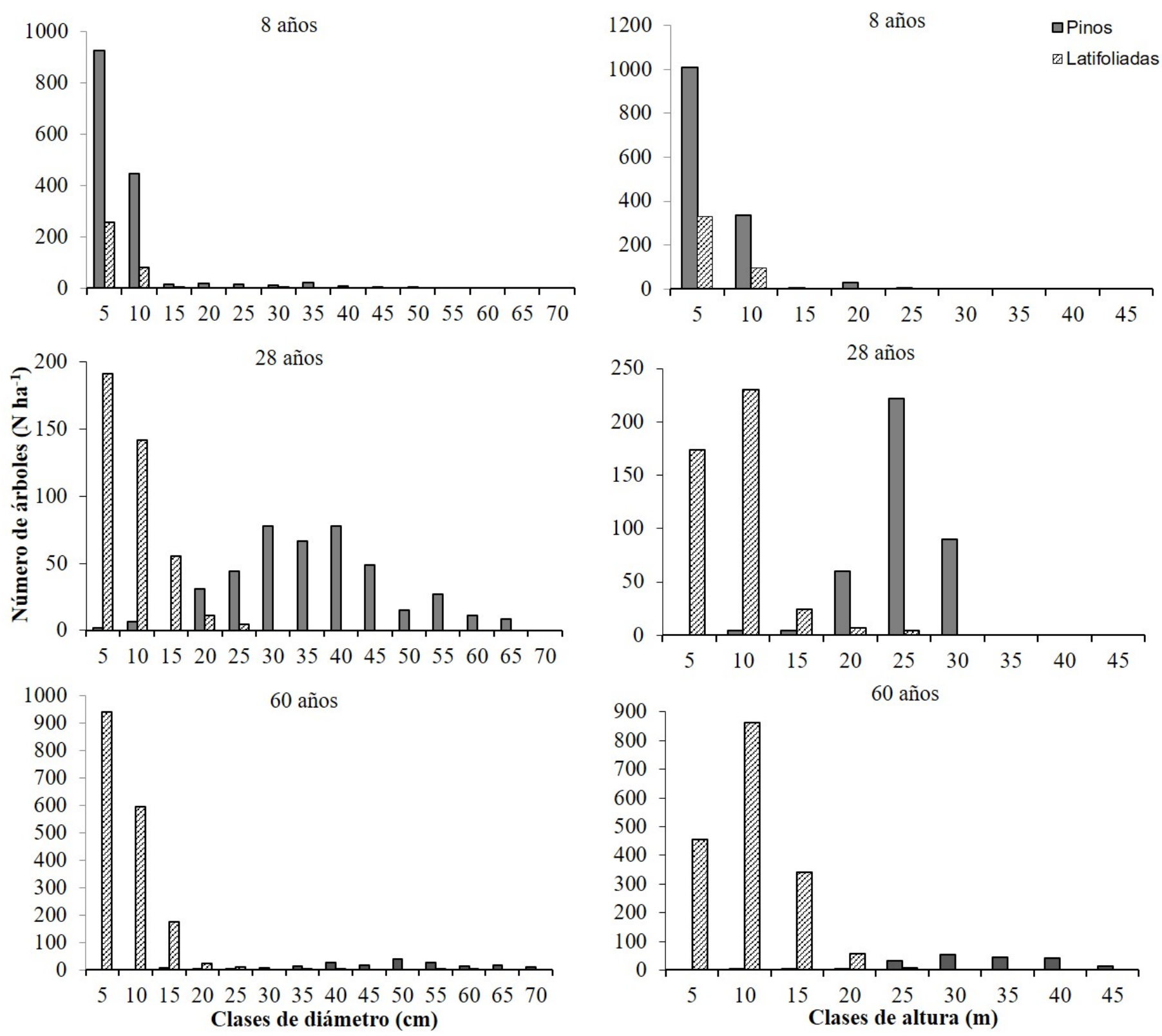

FIGURA 3. Estructura de tamaños (tallos con $\mathrm{D}_{1.30} \geq 2.5 \mathrm{~cm}$ ) en la cronosecuencia postincendio de bosques dominados por Pinus douglasiana en el centro-occidente de México.

A la izquierda se muestra la distribución de diámetros y a la derecha la distribución de alturas. Nótese que las escalas del eje Y son diferentes.

\section{Composición y diversidad de especies}

Se registraron 50 especies arbóreas de 41 géneros y 32 familias; solo tres especies fueron pinos (Pinus douglasiana, Pinus herrerae Martinez y Pinus oocarpa Schiede ex Schltdl (Suplemento 1). La composición del estrato arbóreo estuvo dominada por $P$. douglasiana a través de la cronosecuencia, pero su valor de importancia (IVI) disminuyó con el tiempo postincendio, pasando de 58.3 a 32.1 entre los 8 años y los
60 años de edad, mientras que el VI de las especies latifoliadas aumentó (Tabla 1; Suplemento 1). En los rodales de 8 años, las especies que le siguieron en valor de importancia a $P$. douglasiana, fueron aquellas que tienen capacidad de rebrotar después de incendios, como Morella cerifera (L.) Small, Quercus nixoniana S. Valencia y LozadaPérez, Arbutus xalapensis Kunth (Tabla 1) y arbustos altos como Baccharis heterophylla Kunth, Podachaenium eminens 
(Lag.) Sch.Bip y Monnina xalapensis Kunth, que únicamente se encuentran en etapas tempranas de la sucesión secundaria.

En los rodales de 28 años, las especies con mayor valor de importancia después de $P$. douglasiana fueron $P$. herrerae $(\mathrm{VI}=8.4)$, y especies que exceptuando a $A$. xalapensis $\mathrm{y}$ Viburnum hartwegii Benth, forman parte del dosel del bosque mesófilo de montaña que predomina en las hondonadas y barrancos húmedos, como Clethra fragrans L. M. González y R. Ramírez, Fraxinus ubdei (Wenz.) Lingelsh e Ilex brandegeana Loes.

En los rodales de 60 años, las especies que le siguen en orden de importancia a P. douglasiana fueron Cornus disciflora DC., Myrsine juergensenii (Mez) Ricketson y Pipoly, Carpinus tropicalis Furlow. y Persea bintoni C. K. Allen. (VI > 5), las cuales son también componentes del bosque mesófilo de montaña (Tabla 1).

La similitud en la composición de especies disminuyó con el tiempo postincendio: los rodales de 8 años compartieron 38\% y 13\% de las especies con los rodales de 28 años y 60 años, respectivamente, mientras que estos últimos compartieron entre sí $27 \%$. La similitud de la composición de especies entre rodales de la misma edad parece indicar que esta se incrementa conforme avanza la sucesión, ya que entre los rodales de 8 años varió entre $25 \%$ y $50 \%$, mientras que, tanto los rodales de 28 años como los de 60 años, compartieron entre sí alrededor de 50\% de las especies.

La riqueza y los índices de diversidad de especies arbóreas aumentaron con el tiempo postincendio. La riqueza de especies arbóreas fue de 18, 33 y 31 para los rodales de 8 años, 28 años y 60 años, respectivamente. El análisis de rarefacción mostró que a un mismo nivel de densidad de individuos (381 árboles, densidad menor) el rodal con 28 años presentó la mayor riqueza, seguido por los rodales con 60 años y 8 años de edad (33, 24 y 13 especies, respectivamente; Fig. 4). El índice de diversidad de Shannon $\left(H^{\prime}\right)$ fue menor en los rodales de 8 años, con un valor de 1.1 (IC 95\% = 0.9-1.2), con un incremento a 2.4 y 2.6 en los rodales con 28 años y con 60 años, respectivamente (IC 95\% = 1.7-2.8 y 2.1-2.8; Fig. 5). El índice de Simpson (1-D) tuvo un valor de 0.42 en los rodales con 8 años (IC 95\% $=0.39-0.47$ ), mientras que en los rodales de 28 años y de 60 años fueron de 0.79 y 0.90 (IC $95 \%=0.66-0.89$ y $0.82-0.94$; Fig. 5).

TABLA 1. Índice de Valor de importancia de las 10 especies más importantes en cada clase de edad de la cronosecuencia postincendio en bosques dominados por Pinus douglasiana en el centro-occidente de México.

\begin{tabular}{|c|c|c|c|c|c|}
\hline \multirow{2}{*}{$\begin{array}{l}8 \text { años } \\
\text { Especies }\end{array}$} & \multirow[b]{2}{*}{ VI } & \multicolumn{2}{|l|}{28 años } & \multicolumn{2}{|l|}{60 años } \\
\hline & & Especies & VI & Especies & VI \\
\hline Pinus douglasiana & 58.3 & Pinus douglasiana & 45.2 & Pinus douglasiana & 32.1 \\
\hline Morella cerifera & 6.2 & Pinus herrerae & 8.4 & Cornus disciflora & 9.5 \\
\hline Quercus nixoniana & 5.6 & Clethra fragrans & 4.1 & Myrsine juergensenii & 7.6 \\
\hline Arbutus xalapensis & 5.6 & Fraxinus uhdei & 3.3 & Carpinus tropicalis & 5.7 \\
\hline Pinus herrerae & 2.6 & Viburnum hartwegii & 3.2 & Persea hintonii & 5.0 \\
\hline Baccharis heterophylla & 2.5 & Ilex brandegeana & 2.8 & Magnolia Iltisiana & 4.5 \\
\hline Podachaenium eminens & 2.4 & Arbutus xalapensis & 2.5 & Clethra fragrans & 4.1 \\
\hline Monnina xalapensis & 2.4 & Myrsine juergensenii & 2.1 & Symplocos citrea & 3.1 \\
\hline Alnus jorullensis & 2.3 & Zinowiewia concinna & 2.1 & Ilex brandegeana & 2.9 \\
\hline Quercus obtusata & 1.7 & Ternstroemia lineata & 2.1 & Fraxinus uhdei & 2.6 \\
\hline
\end{tabular}




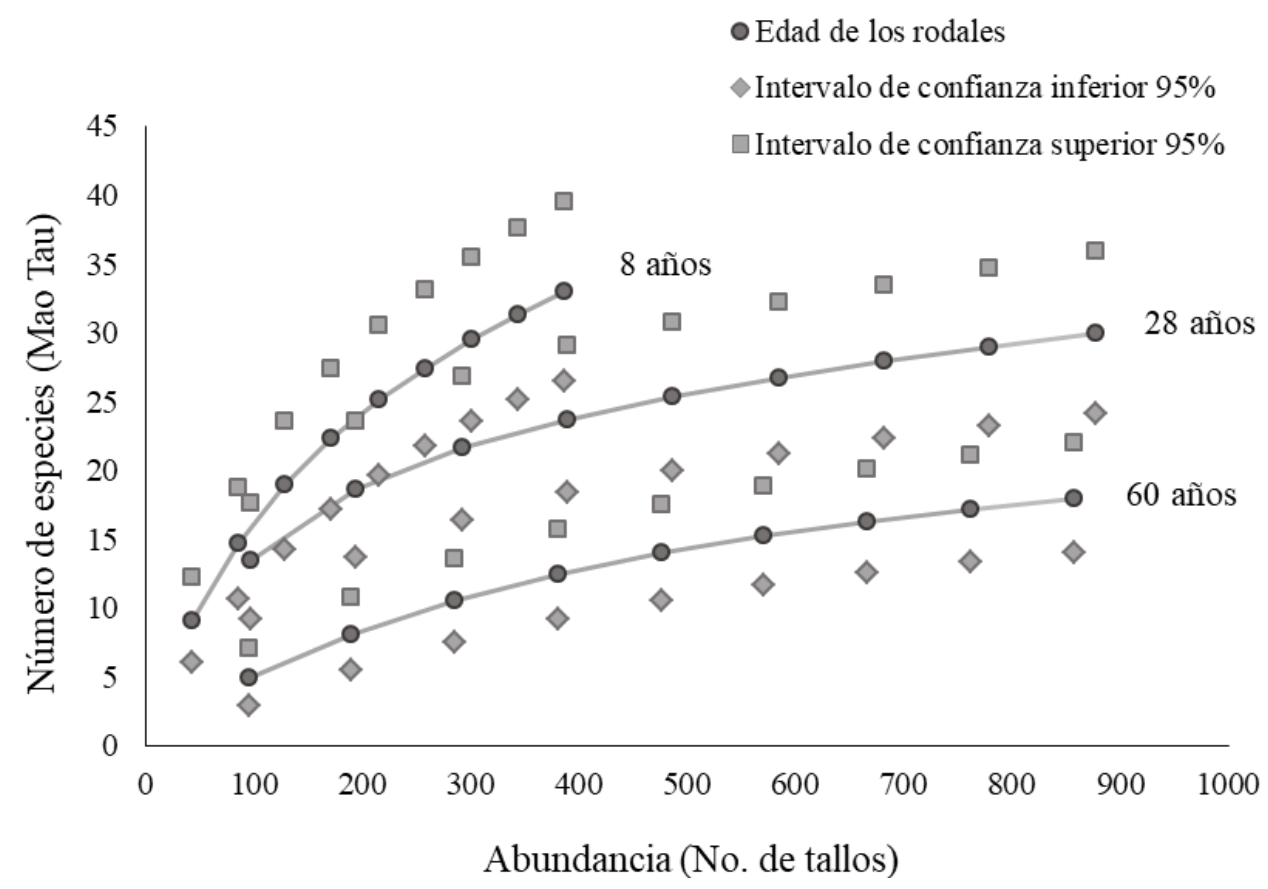

FIGURA 4. Curva de rarefacción para la riqueza de especies a un mismo nivel de abundancia (381 árboles), en la cronosecuencia postincendio de bosques dominados por Pinus douglasiana en el centrooccidente de México.

\section{Carga de combustibles superficiales}

La acumulación de combustibles superficiales presentó cambios marcados a través de la cronosecuencia (Tabla 2). Entre los 8 años y los 28 años de edad, las cargas de HF y HD aumentaron más de cuatro veces en el primer caso y cerca de siete veces en el segundo; a los 60 años, HF disminuyó y HD aumentó ligeramente (Fig. 6a). Las diferencias fueron significativas tanto para $\operatorname{HF}\left(F_{2,26}=20.3\right.$; $P<0.001)$ como para HD $\left(F_{2,26}=31.9 ; P<0.001\right)$. El material leñoso caído, tanto el fino $(<7.6 \mathrm{~cm})$ como el grueso (Fig. 6b), mostraron un patrón inverso y disminuyeron significativamente con el tiempo postincendio $\left(F_{2,26}=10.1 ; P=0.001\right.$ y $F_{2,26}=19.3 ; P<$ 0.001 , respectivamente).

\section{DISCUSIÓN}

Los cambios observados a través de la cronosecuencia muestran que los bosques de $P$. douglasiana del área de estudio se regeneran rápidamente después de incendios de remplazo de rodal. El área basal de los rodales de 8 años fue cuatro y cinco veces menor que en los rodales de 28 años y 60 años, lo que es un indicador de la magnitud de la severidad del fuego (Bartels, Chen, Wulder y White, 2016), y cuya disminución es similar a la hallada en otros estudios (Keyser, et al., 2008; Adámek, Hadincova y Wild, 2016). En los rodales con 28 años postincendio, el área basal se recuperó alcanzando un valor cercano al de los rodales de 60 años y del mismo orden que el área basal promedio de los bosques mixtos de pino-latifoliadas del conjunto del área de estudio (46.6 $\mathrm{m}^{2} \mathrm{ha}^{-1} \pm 0.3 \mathrm{~m}^{2} \mathrm{ha}^{-1}$; Cuevas-Guzmán y Jardel-Peláez, 2004).

Estos datos indican un tiempo corto de regeneración en las condiciones de clima templado-cálido-húmedo del área de estudio, en comparación con bosques de pinos y otras coníferas de climas templado-fríos de Europa (Adámek, Hadincova y Wild, 2016), Canadá (Brassard y Chen 2006; Bartels et al., 2016) o Estados Unidos (Keyser et al., 2008). Los resultados sugieren que los bosques templado-cálidos y húmedos de la Sierra de Manantlán presentan una alta productividad primaria neta que favorece una rápida recuperación de la vegetación después de incendios. 

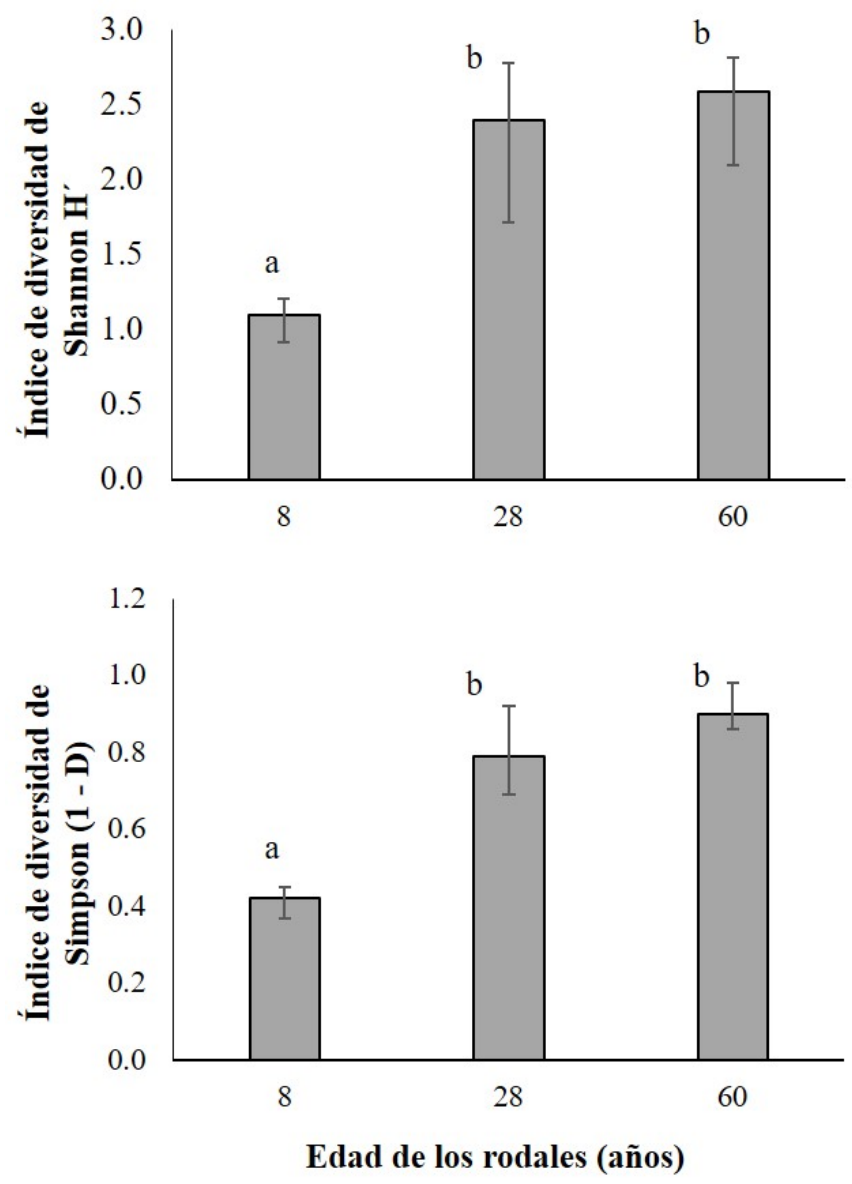

FIGURA 5. Índices de diversidad de Shannon $\left(\mathrm{H}^{\prime}\right)$ y de Simpson (1-D) con sus intervalos de confianza a $95 \%$ en la cronosecuencia postincendio de bosques dominados por Pinus douglasiana en el centro-occidente de México.

Medias con letras comunes no son significativamente diferentes $(P>0.05)$.

\section{Cambios estructurales}

Ocho años después del incendio, los rodales muestran una estructura caracterizada por una alta densidad de árboles con diámetros pequeños $(\leq 10 \mathrm{~cm})$ y baja altura $(\leq 10 \mathrm{~m})$ que corresponden a la regeneración postincendio y algunos árboles grandes vivos que sobrevivieron al fuego; una estructura similar se ha registrado en bosques boreales y templados (Brassard y Chen, 2006; Bartels et al., 2016).

A 28 años del incendio, disminuyó la densidad de tallos de los pinos, pero se incrementó su área basal, lo cual es característico de la fase de autoaclareo en las cohortes de árboles intolerantes a la sombra en etapas tempranas de la sucesión (Oliver y Larson, 1990). Un aspecto remarcable en el área de estudio es que la fase de establecimiento de especies arbóreas tolerantes a la sombra, que forman un subdosel en etapas sucesionales intermedias, de acuerdo con el modelo de Oliver y Larson (1990), ocurre rápidamente y se observa ya en los rodales de 28 años. En los rodales de 60 años, se observó un subdosel de latifoliadas bien establecido. Este patrón sucesional, ha sido documentado en otros trabajos en el área de estudio (Jardel-Peláez 1991, 2008) y otras partes de México (González-Tagle, Schwendenmann, Jiménez-Pérez y Schulz, 2008). Los pinos producen semillas abundantes que se dispersan a distancia, crecen rápidamente y son intolerantes a la sombra, dominan en las etapas iniciales e intermedias de la sucesión. Con el incremento en la edad de los rodales y en ausencia de incendios superficiales, se observa un aumento en la abundancia de las latifoliadas tolerantes a la sombra de los pinos y la formación de un subdosel (Oliver y Larson, 1990).

\section{Diversidad de especies}

La riqueza y diversidad de especies arbóreas aumentó con el tiempo postincendio. Ocho años después del disturbio la riqueza de especies arbóreas fue menor que en las clases de 28 años y 60 años, debido a que la mayoría de las latifoliadas del área de estudio no resisten o toleran los efectos del fuego. El número de especies registrado es comparable a lo observado en otros bosques de pino-encino de México (Alanís-Rodríguez et al., 2012). La diversidad de especies arbóreas aumentó con el tiempo postincendio y alcanzó valores similares entre los rodales de 28 años y 60 años, pero los primeros mostraron una mayor riqueza de especies en el análisis de rarefacción.

Los resultados sugieren que los bosques de Pinus douglasiana se recuperan relativamente rápido en los claros de 2 ha a 40 ha abiertos por incendios. Los elementos claves que incrementan la capacidad de los bosques para recuperar y mantener su estructura y composición después de los incendios, son el tamaño relativamente pequeño de los claros, la sobrevivencia en estos de árboles adultos y el aporte de diásporas desde los rodales circundantes, como se ha demostrado en otros estudios (Keyser et al., 2008; Savage y Mast, 2005). 
TABLA 2. Variación de la carga de combustibles $\left(\mathrm{Mg} \mathrm{ha}^{-1}\right)$ por estrato en la cronosecuencia postincendio en bosques dominados por Pinus douglasiana en el centro-occidente de México.

\begin{tabular}{|c|c|c|c|}
\hline \multirow{2}{*}{ Estrato de la cama de combustibles } & \multicolumn{3}{|c|}{ Tiempo postincendio (años) } \\
\hline & 8 & 28 & 60 \\
\hline Capa de hojarasca fresca & $4.2 \pm 0.6^{a}$ & $18.3 \pm 2.5^{b}$ & $12.3 \pm 0.8^{c}$ \\
\hline Capa de hojarasca en descomposición & $6.2 \pm 1.2^{b}$ & $40.5 \pm 2.0^{a}$ & $42.6 \pm 4.8^{a}$ \\
\hline Hojarasca (total) & $10.4 \pm 1.3^{b}$ & $58.8 \pm 5.2^{a}$ & $54.9 \pm 4.7^{a}$ \\
\hline$<0.06 \mathrm{~cm}(1 \mathrm{~h})$ & $0.4 \pm 0.1^{a}$ & $0.2 \pm 0.05^{a}$ & $0.4 \pm 0.2^{a}$ \\
\hline $0.06 \mathrm{~cm}-2.5 \mathrm{~cm}(10 \mathrm{~h})$ & $5.3 \pm 1.8^{\text {a }}$ & $2.8 \pm 0.6^{a}$ & $2.5 \pm 0.3^{a}$ \\
\hline $2.6 \mathrm{~cm}-7.6 \mathrm{~cm}(100 \mathrm{~h})$ & $12.0 \pm 1.4^{b}$ & $4.2 \pm 0.7^{a}$ & $3.7 \pm 0.5^{a}$ \\
\hline Material leñoso caído fino (total) & $17.8 \pm 3.1^{\mathrm{b}}$ & $7.2 \pm 1.2^{a}$ & $6.6 \pm 0.9^{a}$ \\
\hline Material leñoso caído grueso $>7.6 \mathrm{~cm}(1000 \mathrm{~h})$ & $79.5 \pm 10.1^{\mathrm{b}}$ & $17.9 \pm 3.8^{\mathrm{a}}$ & $18.4 \pm 2.2^{\mathrm{a}}$ \\
\hline
\end{tabular}

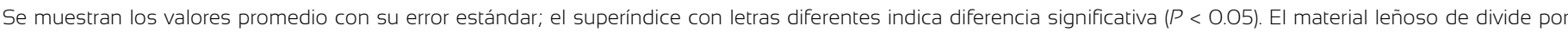
tamaño de las partículas y entre paréntesis se indica la clase de tiempo de retardo o respuesta a la humedad ambiente.

\section{Acumulación de combustibles superficiales}

El cambio en la carga de combustibles superficiales (hojarasca y material leñoso caído ligero) a través de la cronosecuencia, indica un aumento del potencial de incendios superficiales con el tiempo postincendio. Mientras que en los rodales de 8 años la carga de combustible superficial ligero (hojarasca y material leñoso caído fino $<7.6 \mathrm{~cm}$ de diámetro) fue en promedio de 28.2 $\mathrm{Mg}$ ha-1 ${ }^{-1}$ en los rodales de 28 años y 60 años alcanzó valores de 66.0 Mg ha-1 y $61.5 \mathrm{Mg} \mathrm{ha}^{-1}$ (Tabla 2). La magnitud de esta diferencia en el potencial de incendios superficiales puede apreciarse haciendo una simulación en el programa BehavePlus 5.0 (Heinsch y Andrews, 2010), utilizando modelos de combustible (Scott y Burgan, 2006) análogos a las condiciones de carga de hojarasca registradas en el área de estudio (modelo TL3 para los rodales de 8 años y TL9 para los de 28 años y 60 años). Bajo las mismas condiciones de humedad de los combustibles $(6 \%-8 \%$ de humedad para combustibles superficiales ligeros y $60 \%-90 \%$ para hierbas y arbustos), velocidad del viento $\left(6 \mathrm{~km} \mathrm{~h}^{-1}\right)$ e inclinación de la pendiente (5\%), la velocidad de propagación del fuego cambia de $1.3 \mathrm{~m} \mathrm{~s}^{-1}$ a $4.1 \mathrm{~m} \mathrm{~s}^{-1}$ y la longitud de la llama de $0.7 \mathrm{~m}$ a $1.7 \mathrm{~m}$ entre los rodales de 8 años (carga moderada de combustible superficial) y de 28 años y 60 años (carga alta de combustibles superficiales).

La carga de material leñoso caído grueso fue significativamente mayor en los rodales de 8 años que en los de 28 años y 60 años, debido al efecto de mortalidad de árboles durante los incendios del año 2003. Estudios que han cubierto cronosecuencias más largas, muestran un patrón general en forma de " $U$ " en la variación temporal de la biomasa de material leñoso caído grueso: las cargas son altas después de incendios de remplazo de rodal, disminuyen en etapas intermedias de la sucesión y aumentan en rodales de viejo crecimiento (Harmon y Chen, 1991). Sin embargo, la ignición del material leñoso caído grueso depende de la intensidad del calor generado por la combustión de la hojarasca, que es el componente del complejo de combustibles que controla la iniciación y propagación de los incendios superficiales (Pyne et al., 1996).

La capa de hojarasca en descomposición, cuya carga aumentó marcadamente entre los 8 años y los 28 años y siguió incrementándose ligeramente hasta los 60 años de edad de los rodales, es más densa que la capa de hojarasca fresca y representó más del doble de la biomasa de esta 

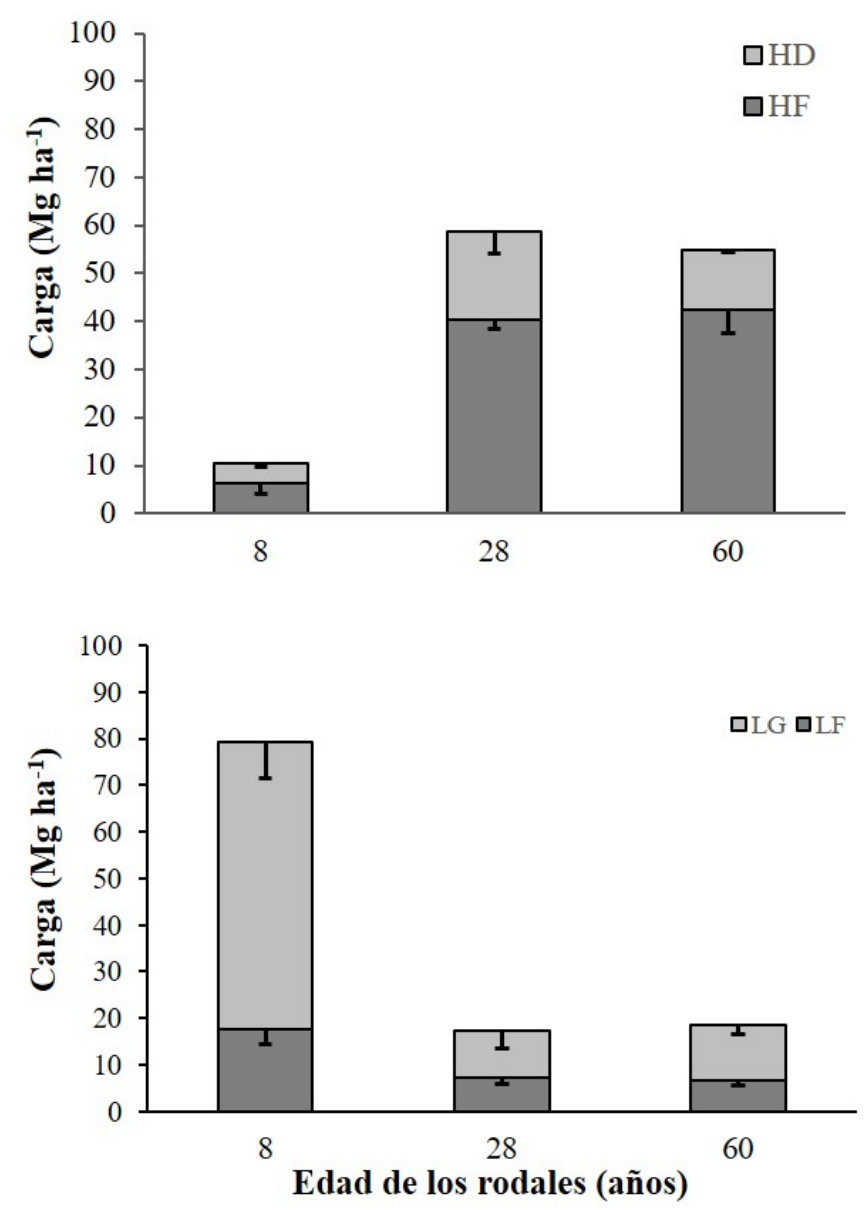

FIGURA 6. Carga de combustibles superficiales (hojarasca y material leñoso caído), a través de la cronosecuencia postincendio en bosques dominados por Pinus douglasiana en el centro-occidente de México.

$H F$, hojarasca fresca; $H D$, hojarasca fragmentada; $L F$, material leñoso fino (diámetro $<7.6 \mathrm{~cm}$ ) y LG, material leñoso grueso $(>7.6 \mathrm{~cm}$ de diámetro). Las barras son valores promedio de cada categoría con su error estándar.

última; cuando está seca y se enciende, genera más calor y aumenta el tiempo de residencia del fuego, incrementando a su vez la intensidad del incendio, lo cual puede causar la mortalidad de árboles del dosel y la formación de claros, como se ha observado en el área de estudio.

Comparando los resultados obtenidos con los de otro trabajo realizado en el área de estudio (Alvarado et al., 2008), las cargas de hojarasca en los rodales de 28 años y de 60 años (Tabla 2), fueron del mismo orden que las de un rodal de aproximadamente 45 años al tiempo de la medición en el estudio citado (58.2 Mg ha-1 , sitio BMS 09). En contraste, otro sitio observado en el mismo estudio, donde se formó un claro después de un incendio severo, tenía a los 7 años una carga de $16.9 \mathrm{Mg} \mathrm{ha}^{-1}$ de hojarasca, mayor que en el rodal de 8 años del presente trabajo, pero la mitad de la carga de material leñoso caído (45.9 $\mathrm{Mg} \mathrm{ha}^{-1}$, sitio BMS 03). Con respecto a la carga de material leñoso caído, esta fue menor en los rodales de 28 años y de 60 años (alrededor de $18 \mathrm{Mg} \mathrm{ha}^{-1}$ ), que en rodales densos (40 años - 50 años), donde habían ocurrido incendios superficiales 16 años y 19 años antes de la medición realizada por Alvarado et al. (2008), en los cuales se señalan entre $31.6 \mathrm{Mg} \mathrm{ha}^{-1}$ y $38.0 \mathrm{Mg}$ ha $^{-1}$ (sitios BMS 08 y BMS 09). La mayor cantidad de material leñoso caído en estos rodales puede deberse a la mortalidad de árboles por competencia en rodales densos en la fase sucesional de autoaclareo (Oliver y Larson, 1990).

En la ECLJ la supresión del fuego combinada con quemas prescritas y la tolerancia a la propagación de incendios forestales en ciertas áreas, ha sido planteada como un experimento dirigido a recuperar la cobertura de bosque mixto de pino-latifoliadas y bosque mesófilo de montaña (Jardel, 2008), al mismo tiempo que se mantiene la heterogeneidad del mosaico del paisaje incluyendo áreas quemadas con distinto grado de severidad y tiempo postincendio, de lo cual depende el mantenimiento de hábitat para las especies y comunidades de plantas pirófilas y de etapas sucesionales tempranas (Cuevas-Guzmán y Jardel Peláez et al., 2004).

\section{CONCLUSIONES}

En bosques dominados por $P$. douglasiana en el centrooccidente de México ocurrieron incendios severos de pequeña extensión (1 ha a 40 ha), en los cuales, ocho años después, atributos como el área basal, la riqueza y la diversidad de especies arbóreas son menores comparados con rodales de 28 años y de 60 años. Sin embargo, se observa una recuperación rápida de dichos atributos, ya que 28 años después del incendio los rodales afectados alcanzaron una estructura y diversidad de la comunidad arbórea similar a los rodales con 60 años de edad, resultados que respaldan la hipótesis planteada. Condiciones de sitios como el tamaño de los claros abiertos por el incendio y la 
sobrevivencia de individuos adultos a los incendios, así como condiciones ambientales de temperatura y precipitación características de estos bosques de zonas templadas cálidas y húmedas, parecen favorecer altas tasas de productividad primaria y una recuperación más rápida de la vegetación después de incendios. Conforme incrementa el tiempo después del disturbio ecológico, se observó una acumulación de combustibles finos cuatro veces mayor en los rodales de 28 años y de 60 años, con respecto a los rodales de 8 años. La alta carga de hojarasca modifica el comportamiento potencial del fuego superficial y podría llegar a sostener la propagación de nuevos incendios superficiales intensos.

\section{RECONOCIMIENTOS}

El estudio fue financiado por el Consejo Estatal de Ciencia y Tecnología del estado de Jalisco, como parte del proyecto "Estructura, diversidad y reservorios de carbono de bosques de cañada en el Pacífico Mexicano” (PS-2009-664).

\section{REFERENCIAS}

Adámek, M., Hadincova, V., \& Wild, J. (2016). Long-term effect of wildfires on temperate Pinus sylvestris forests: Vegetation dynamics and ecosystem resilience. Forest Ecology and Management, 380, 285-295. doi: 10.1016/j.foreco.2016.08.051.

Agee, J. K. (1993). Fire ecology of Pacific Northwest forests. Washington, Estados Unidos: Island Press.

Agee, J. K. (1998). Fire and pine ecosystems. En M. S. Richardson (Ed.), Ecology and Biogeography of Pinus (pp. 193-218). Cambridge, Reino Unido: Cambridge University Press.

Alanís-Rodríguez, E., Jiménez-Pérez, J., Valdecantos-Dema, A., González-Tagle, M. A., Aguirre-Calderón, O. A., \& Treviño-Garza E. J. (2012). Composición y diversidad de la regeneración natural en comunidades de Pinus-Quercus sometidas a una alta recurrencia de incendios en el noreste de México. Revista Mexicana de Biodiversidad, 83(4), 1208-1214. doi: $10.7550 / \mathrm{rmb} .29708$

Alvarado, E. C., Morfín-Ríos, J. E., Jardel-Peláez, E. J., Vihnanek, R. E., Wright, D.K., Sand- berg, D. V., Michel-Fuentes, J. M., Wright, C. S., Ottmar, R. D., Sandberg, D. V., \& NájeraDíaz, A. (2008). Fotoseries para la cuantificación de combustibles forestales de México: bosques montanos subtropicales de la Sierra Madre del Sur y bosques templados y matorral submontano del norte de la Sierra
Madre Oriental. Recuperado de: http://www.fs.fed.us/pnw/fera/publications/fulltext/Pho toSeriesMexicoUW-FERAPublication.pdf

Bartels, S. F., Chen, H. Y. H., Wulder, M. A., \& White, J. C. (2016). Trends in post-disturbance recovery rates of Canada's forests following wildfire and harvest. Forest Ecology and Management, 361, 194-207. doi: 10.1016/j.foreco.2015.11.015.

Bowman, D. M. J. S., Murphy, B. P., Boer, M. M., Bradstock, R. A., Cary, G. J., Cochrane, M. A., Fensham, R. J., Krawchuk M. A., Price, O. F., \& Williams, R. J. (2013). Forest fire management, climate change, and the risk of catastrophic carbon losses. Frontiers in Ecology and the Environment, 11(2), 66-68. doi:10.1890/13.WB.005.

Brassard, B. W., \& Chen, H. Y. H. (2006). Stand structural dynamics of North American boreal forests. Critical Reviews in Plant Sciences, 25 (2), 115-137. doi:10.1080/07352680500348857.

Cerano-Paredes, J., Villanueva-Díaz, J., Cervantes-Martínez, R., Fulé, P. Z., Yocom, L. Esquivel-Arriaga, G., \& JardelPeláez, E. J. (2015). Historia de incendios en un bosque de pino de la sierra de Manantlán, Jalisco, México. Bosque, 36(1), 41-52. doi:10.4067/S0717-92002015000100005.

Cuevas-Guzmán, R., \& Jardel-Peláez, E. J. (Eds.). (2004). Flora y Vegetación de la Estación Científica Las Joyas. Guadalajara, México: Universidad de Guadalajara.

Di Rienzo, J. A., Casanoves, F., Balzarini, M. G., Gonzalez, L., Tablada, M., \& Robledo C. W. (2008). InfoStat, versión 2008, Grupo InfoStat, FCA, Universidad Nacional de Córdoba, Argentina: Universidad Nacional de Córdoba.

Galicia, L., Potvin, C., \& Messier, C. (2015). Maintaining the high diversity of pine and oak species in Mexican temperate forest: a new management approach combining functional zoning and ecosystem adaptability. Canadian Journal of Forestry Research, 45(10), 1358-1368. doi:10.1139/cjfr-20140561.

Gernandt, D. S., \& Pérez-de la Rosa, J. A. (2014). Biodiversidad de Pinophyta (coníferas) en México. Revista Mexicana de Biodiversidad, 85, 126-133. doi:10.7550/rmb.32195.

González-Tagle, M. A., Schwendenmann, L., Jiménez-Pérez, J., \& Schulz, R. (2008). Forest structure and woody plant species composition along a fire chronosequence in mixed pine-oak forest in the Sierra Madre Oriental, Northeast Mexico. Forest Ecology and Management, 256, 161-167. doi: 10.1016/j.foreco.2008.04.021. 
Harmon, M. E., \& Chen, H. (1991). Coarse Woody Debris Dynamics in Two Old-Growth Ecosystems. BioScience, 41(9), 604-610. doi:10.2307/1311697.

Heinsch, F. A, \& Andrews, P. L. (2010). BehavePlus fire modeling system, version 5.0: design and features. Gen. Tech. Rep. RMRS-GTR-249. Fort Collins, Estados Unidos: US Department of Agriculture, Forest Service, Rocky Mountain Research Station.

Jardel-Peláez EJ. (1991). Perturbaciones naturales y antropogénicas y su influencia en la dinámica sucesional de los bosques de Las Joyas, Sierra de Manantlán, Jalisco. Tiempos de Ciencia, 22, 9-26.

Jardel-Peláez, E. J. (2008). Sucesión ecológica y restauración de bosques subtropicales de montaña en la Estación Científica Las Joyas, México. En: M. González-Espinosa, N. RamírezMarcial, J. M. Rey-Benayas (Eds.), Restauración de bosques en América Latina (pp. 77-97). México D.F: Fundación Internacional para la Restauración de Ecosistemas y MundiPrensa.

Jardel-Peláez, E. J., Pérez-Salicrup, D., Alvarado-Celestino, E., \& Morfín-Ríos, J. E. (2014). Principios y criterios para el manejo del fuego en ecosistemas forestales. Zapopan, México: Comisión Nacional Forestal.

Keyser, T. L., lentil, L. B., Smith, F. W., \& Shepperd, W. D. (2008). Changes in forest structure following a mixedseverity wildfire in ponderosa pine forests of the Black Hills, South Dakota, Estados Unidos. Forest Science, 54(3), 328-338.

Magurran, A, E. (2004). Measuring biological diversity. Massachusetts, Estados Unidos: Blackwell Science.

McCune, B., \& Mefford, M. J. (2006). PC-ORD. Multivariate Analysis of Ecological Data. Version 5.10. Gleneden Beach, Estados Unidos: MjM Software.

Morfín-Ríos, J. E., Jardel-Peláez, E. J. Michel-Fuentes, J. M., Alvarado-Celestino, E. (2012). Caracterización y cuantificación de combustibles forestales. Guadalajara, México: Comisión Nacional Forestal-Editorial Universidad de Guadalajara.

Odion, D. C., Hanson, C. T. Arsenault, A., Baker, W. L., DellaSala, D. A., Hutto, R. L., Klenner, W., Moritz, M. A., Sherriff, R. L., Veblen, T. T., \& Williams, M. A. (2014). Examining historical and current mixed-severity fire regimes in ponderosa pine and mixed-conifer forests of Western
North America. PLoS ONE, 9(2), e87852. doi:10.1371/journal.pone.0087852.

Oliver, C. D., \& Larson, B. C. (1990). Forest stand dynamics. New York, Estados Unidos: McGraw Hill.

Pyne, S. J., Andrews, P. L., \& Laven, R. D. (1996). Introduction to Wildland Fire. New York, Estados Unidos: John Wiley y Sons.

Rodríguez-Trejo, D., \&. Fulé, P. (2003). Fire ecology of Mexican pines and a fire management proposal. International Journal of Wildland Fire, 12(1), 23-37. doi:10.1579/0044-744737.7.548.

Savage, M., \& Mast, J. N. (2005). How resilient are southwestern ponderosa pine forest after crown fires?. Canadian Journal Forest Research, 35, 967-977. doi: 10.1139/x05-028.

Schulze, E. D., Wirth, C., \& Heimann, M. 2000. Managing forests after Kyoto. Science 289(5487): 2058-2059. doi:10.1126/science.289.5487.2058.

Scott, J. H., \& Burgan, R. E. (2005). Standard fire behavior fuel models: a comprehensive set for use with Rothermel's surface fire spread model. Gen. Tech. Rep. RMRS-GTR153. Fort Collins, Estados Unidos. Recuperado de https://www.fs.usda.gov/treesearch/pubs/9521.

Van Wagner, C. E. (1982). Practical aspects of the line intersect method (Report PI-X-12). Chalk River, Canada: Canadian Forestry Service-Petawawa National Forestry Institute.

Manuscrito recibido el 28 de noviembre de 2018

Aceptado el 20 de febrero de 2019

Publicado el 13 de diciembre de 2019

Este documento se debe citar como:

Quintero-Gradilla, S. D., Jardel-Peláez, E. J., Cuevas-Guzmán, R., GarcíaOliva, F., \& Martínez-Yrizar, A. (2019). Cambio postincendio en la estructura y composición del estrato arbóreo y carga de combustibles en un bosque de Pinus douglasiana de México. Madera y Bosques, 25(3), e2531888. doi: 10.21829/myb.2019.2531888

Madera y Bosques por Instituto de Ecología, A.C. se distribuye bajo una Licencia Creative Commons Atribución-NoComercialCompartirlgual 4.0 Internacional. 